\title{
Nano Electrospray Combined with a Quadrupole Ion Trap for the Analysis of Peptides and Protein Digests*
}

\author{
Roman Körner, Matthias Wilm, Kenneth Morand, ${ }^{\dagger}$ Michael Schubert, \\ and Matthias Mann \\ Protein and Peptide Group, European Molecular Biology Laboratory, Heidelberg, Germany
}

\begin{abstract}
A nano electrospray (NanoES)/ion trap combination was developed to take advantage of the long spraying time of the NanoES source (Wilm, M. S.; Mann, M. Int. J. Mass Spectrom. Ion Processes 1994, 136, 167-180) for extensive experiments on the ion trap. The low flow rate associated with the NanoES allows for optimization of experimental conditions and data acquisition for more than $30 \mathrm{~min}$ with $1 \mu \mathrm{L}$ of solution. Thus, even time-consuming experiments, such as multiple fragmentation steps or the sequencing of many components in a mixture, can be performed with very small volumes of sample. Stored waveform inverse Fourier transformation (SWIFT) signals were used during the injection period to accumulate ions of low intensity and to improve the dynamic range of the quadrupole ion trap. To sequence peptides, a combination of nozzle-skimmer fragmentation, SWIFT accumulation of a single fragment to high intensity, and a second fragmentation step was employed to obtain complete sequence information. Unseparated peptide mixtures were infused and weak portions of the spectrum were enhanced by using the SWIFT method, followed by fragmentation of various accumulated peptide ions. (J Am Soc Mass Spectrom 1996, 7, 150-156)
\end{abstract}

I addition to well established types of mass spectrometers, such as magnetic sector instruments, time-of-flight, and triple quadrupole mass spectrometers, quadrupole ion traps are now commercially available for the analysis of biomolecules. Potential advantages of the ion trap are its high resolving power and MS" capability, its compact size, and modest price.

Originally the ion trap was a low-performance mass spectrometer used mainly for gas chromatography-mass spectrometry analysis of low molecular weight compounds. During the last decade resolving power, mass range, and sensitivity have been increased dramatically through extensive work by such researchers as Cooks, McLuckey, and the group at Finnigan-MAT [1, 2]. Resolving power in the ion trap can be increased by decreasing the scan speed of the instrument-albeit at the cost of longer analysis time. Recent work has demonstrated resolving power that exceeds one million $[3,4]$. The development of resonance ejection techniques has extended the possible mass-to-charge ratio of the ion trap to $50,000 \mathrm{u}$ per

Address reprint requests to Dr. Matthias Mann, Protein and Peptide Group, European Molecular Biology Laboratory (EMBL), Meyerhofstrasse 1, D-69012 Heidelberg, Germany.

* Presented at the 43rd ASMS Conference.

'Present address: The Procter and Gamble Co., Cincinnati, Ohio, USA.

${ }^{\ddagger}$ Bruker-Franzen Analytik GmbH, Bremen, Germany.

charge [5] and the tandem mass spectrometry capabilities of the trap now include many successive fragmentation steps [6]. Franzen [7] and Wang [8] investigated nonlinear resonances to improve scan speed and resolving power. Furthermore, Cooks and co-workers [5] have shown that the ion trap is capable of subfemtomole sensitivity. However, all of these high-performance characteristics need considerable measurement time for optimization of the signal. Therefore, it is desirable to couple the ion trap to a source that provides ion current for a considerable time.

The ion trap previously has been interfaced to an clectrospray source-first by the research group at Oak Ridge National Laboratory [9]. To provide long spraying times and efficient use of sample we equipped an ion trap mass spectrometer with the nano electrospray (NanoES) source developed by our group and used routinely on a triple quadrupole instrument in our laboratory. The NanoES source basically consists of a finely drawn gold-coated glass capillary placed 1-2 $\mathrm{mm}$ in front of the orifice of the mass spectrometer. The NanoES source requires no forced liquid flow (and hence no pumps) or injection valves. The tip of the glass capillary has an orifice of $1-3 \mu \mathrm{m}$ in diameter, which results in a flow rate of about $20-30 \mathrm{~nL} / \mathrm{min}$, which thus allows measurements for more than $30 \mathrm{~min}$ on $1 \mu \mathrm{L}$ of sample solution. Because the diameter of electrosprayed droplets decreases with flow rate [10], the droplets have a calculated diameter of only $180 \mathrm{~nm}$ 
when they are generated with the NanoES. This compares to droplets in the range of 1-2 $\mu \mathrm{m}$ with conventional electrospray sources with flow rates of $0.5-10$ $\mu \mathrm{L} / \mathrm{min}$. Smaller droplets are preferable because their charge-to-volume ratio is larger, which results in a greater accessibility of the analyte ions for ionization. This in combination with the favorable geometry of the ion source (a larger percentage of analyte ions are transferred into the vacuum system) makes the ionization process more efficient than in conventional systems. Thus, although the NanoES source has an approximately 40 times lower flow rate than conventional electrospray sources, we have not observed a decrease in ion current.

Because of space-charge effects, the number of storable ions is limited in trapping instruments [11, 12]. Therefore, when the level of chemical noise is high, only a small number of the stored ions are analyte ions, which in turn limits sensitivity. However, application of stored waveform inverse Fourier transformation (SWIFT) pulses [13, 14] to one of the ion trap endcaps allows the selective storage of ions of interest in the trap. Thus even low abundance ions can be isolated, accumulated, and investigated.

In this article we present the combination of $\mathrm{Na}$ noES with an ion trap, SWIFT for the selective storage of peptides, and an application to peptide analysis and sequencing. We further use the long measurement time to overcome some of the weaknesses of the ion trap in tandem mass spectrometry mode (such as the low mass cutoff and the predominant population of low energy pathways) through the use of a second fragmentation step.

Most of the separate technologies combined in this investigation have been described before. However, it has not been realized widely that the NanoES-in contrast to conventional electrospray ion sources and to other miniaturized electrospray ion sources $[15,16]$ - effectively removes the time limitation on complex investigations such as multiple fragmentation stages. Such time limitations have been one of the major reasons for the relative lack of practical applications of trapping instruments.

\section{Experimental}

\section{Ion Trap}

All experiments were carried out on a prototype of the Esquire ion trap (Bruker-Franzen Analytic $\mathrm{GmbH}$, Bremen, Germany) [17-19]. Our instrument has a fundamental rf frequency of $691,200 \mathrm{~Hz}$ and a standard mass-to-charge ratio range up to $1750 \mathrm{u}$ per charge. The standard scan uses the nonlinear hexapole resonance at $\mathrm{rf} / 3[7,8]$ for resonance ejection [20] of ions with a scan speed of approximately $1450 \mathrm{u} / \mathrm{s}$. The ion trap was operated at an estimated $\mathrm{He}$ bath gas pressure of $1 \times 10^{-2}$ mbar. A differentially pumped Analytica interface (Analytica of Branford, Inc., Branford,
CT) transfers the ions from the NanoES source to the mass spectrometer.

\section{NanoES Source}

Electrospray needles were made with a micropipette puller (model P-87 puller, Sutter Instrument Company, Novato, USA) from borosilicate capillaries (Clark Electromedical Instruments, Pangbourme Reading, England) and were gold-coated in batches of about 20 in a vapor deposition instrument (SCD 020, Balzers, Wiesbaden, Germany). Several hundred needles can be made in a few hours and a new needle was used for each experiment. Problems with durability of the needles were not encountered during analyses that lasted up to $3 \mathrm{~h}$. The sample solution $(0.5-20 \mu \mathrm{L})$ is loaded into the needle with a gel loader pipette. The gas-tight needle holder (see Figure 1) allows application of an arbitrary air pressure below 1 bar to stabilize the spray; this is especially helpful if the analyte solution contains salt (concentrations up to $0.1 \mathrm{M}$ are tolerated by the device). The needle is positioned 1-2 $\mathrm{mm}$ in front of the orifice. Positioning of the NanoES source and spraying were observed through a Leitz stereomicroscope at sixteenfold magnification (Wild M3B, Leica, Heerbrugg, Switzerland). A voltage of $600 \mathrm{~V}$ is sufficient to start the spray because of the small distance between needle tip and orifice. The orifice size of the glass transfer capillary into the mass spectrometer was reduced from the original $500 \mu \mathrm{m}$ to $200 \mu \mathrm{m}$ without apparent decrease in ion current. The decrease in capillary entrance diameter resulted in an advantageous drop of all the pressures in the interface to about half the previous values. In addition, no $\mathrm{N}_{2}$ counterflow was needed when the smaller orifice was used.

\section{SWIFT}

A computer program was written in the programming language $\mathrm{C}++$ to define the amplitude of the excitation function as a function of frequency. This function is defined by the desired mass-to-charge ratio range (which corresponds to a range of secular frequencies [21] to be isolated in the experiment). The resulting notch ranged between some and several hundred atomic mass units. The frequency domain function was

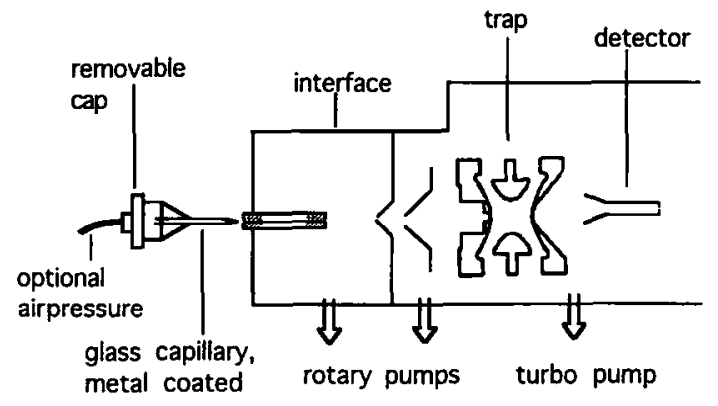

Figure 1. Overview of the NanoES/ion trap instrumental setup. 
transformed to a time domain function by using a fast Fourier transformation algorithm [22, 23] and a quadratic phase modulation [13]. The time domain signal consists of an overlap of the frequencies as defined before. The time domain signal information was then transferred to a waveform generator (Wavetek Model 95, Wavetek Electronics GmbH, Ismaning, Germany) via a general purpose interface bus (GPIB) (Keithley KPC-488.2AT, Keithley Instruments $\mathrm{GmbH}$, Germering, Germany). This waveform generator has a 12-bit digital analog converter (DAC) output and was used with an amplitude of about $11 \mathrm{~V}$ (peak-to-peak) and a sample rate of $2.2 \mathrm{MHz} .8192$ discrete values were transferred to the waveform generator to specify the voltage pulses. These voltage pulses were applied to the grounded ion trap endcap. The $50-\Omega$ output that was used for this task was connected directly to this endcap and a $50-\Omega$ resistor was added between the endcap and ground potential. A software-controled transistor-transistor logic (TTL) level signal that originates in the ion trap electronics and that is adjustable by the user controls the time at which the output of the waveform is applied to the endcap. For the SWIFT experiments described here the waveform output was triggered to activate a train of SWIFT pulses during the entire injection time. This results in the excitation and ejection of all ions by absorption of energy from a voltage signal that matches their secular frequency except for those ions in the small notched window previously specified.

\section{Resonance Fragmentation in the Ion Trap}

For collision-induced dissociation (CID) of the trapped ions an excitation frequency with an amplitude of $350-600 \mathrm{mV}$ (peak-to-peak) was applied for $30 \mathrm{~ms}$. The excitation frequency was adjusted so as to dissociate all isotopes of a given peptide ion. (A range of masses can be dissociated by a single frequency because of frequency line broadening and off-resonance energy absorption.) The amplitude of the excitation frequency was chosen to maximize the ion fragment intensity.

\section{Experiments with Two Fragmentation Steps}

The Analytica interface allows fragmentation of the electrosprayed ions in the first differential pumping stage ( 0.9 mbar) (nozzle-skimmer fragmentation) by increasing the voltage between the end of the capillary and the first voltage lens from between 10 and $100 \mathrm{~V}$ to between 100 and $150 \mathrm{~V}$. Experiments were performed by a first fragmentation of the ion in question in the trap, which thereby determined fragments of interest. In a second experiment the first fragmentation step was performed by nozzle-skimmer dissociation and the trap was filled selectively to the space-charge limit with a single fragment by using suitable SWIFT pulses. This fragment was then further dissociated by CID as described in the preceding text.

\section{Chemicals}

All samples were dissolved in a high-performance liquid chromatography (HPLC) grade mixture of $49 \%$ water, $49 \%$ methanol, and $2 \%$ formic acid. The model peptide HSIAGPPVPPR was synthesized by our group. Leucine enkephalin-Arg and horse heart cytochrome $c$ were purchased from Sigma Chemie $\mathrm{GmbH}$ (Deisenhofen, Germany). Cytochrome $c$ was digested with trypsin $(1: 50 \mathrm{w} / \mathrm{w})$ in a $0.1-\mathrm{M}$ ammonium bicarbonate buffer solution at $37^{\circ} \mathrm{C}$. After $8 \mathrm{~h}$ the digest was dried in a vacuum centrifuge and redissolved in $49 \%$ water, $49 \%$ methanol, and $2 \%$ formic acid solution.

\section{Results and Discussion}

Initially the NanoES/ion trap combination was investigated for suitability for peptide analysis. The setup is easy to operate and use. To load a sample, position the needle, and start the spraying process takes about 1-2 min. Typically the spray is stable during the entire analysis time without any further adjustment of operation parameters. The resolving power with the standard scan speed is sufficient to resolve isotopes of doubly charged peptide ions over the entire mass range [17]. The mass accuracy of our prototype ion trap is approximately $0.2-0.5 \mathrm{u}$ for the entire mass range without the use of internal standards. For practical applications the sensitivity is limited mainly by the level of chemical noise and lies clearly below the picomole level.

\section{SWIFT Performance}

With the SWIFT setup described herein the user can specify a mass-to-charge range of ions to be accumulated. By application of a broad-band excitation voltage during injection time, all ions except the analyte ions immediately are ejected resonantly. Because only the analyte ions are stored the injection time can be prolonged until the space-charge limit is reached for the ion species of interest. Over the course of the injection period the analyte ions are therefore concentrated in the trap.

Figure 2a shows an example of a contaminated peptide sample. (The contamination originated from residues-presumably mainly salts - from the peptide synthesis.) It is evident that the background contributes significantly to the total amount of ions present in the trap and that the ion of interest is not even the base peak of the spectrum. After application of the SWIFT signal, the doubly charged peak clearly is evident with a signal-to-noise increase of more than a factor of 100 (Figure $2 \mathrm{~b}$ ). Note also that the number of trapped ions of interest-at the space-charge limit-increased by 2 orders of magnitude.

Figure 3 shows the application of SWIFT to the low level detection of a peptide. One microliter of a 10$\mathrm{fmol} / \mu \mathrm{L}$ solution of the model peptide leucine 
a

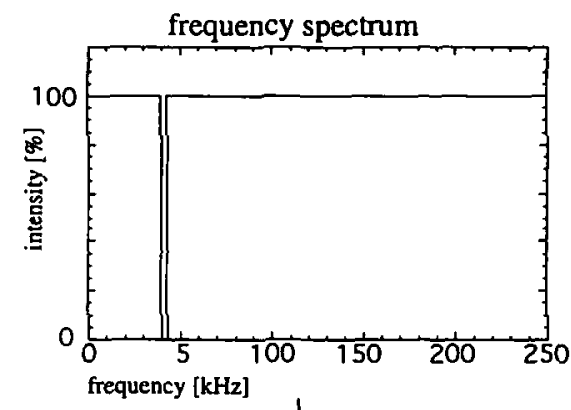

b

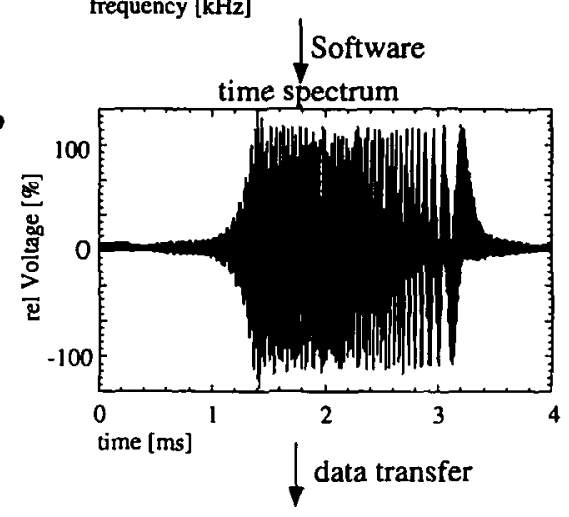

C

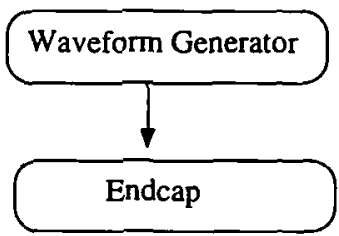

Figure 2. Schematic for the creation of the SWIFT pulses (see text for further explanations).

enkephalin-Arg was loaded into the spray needle and a broad isolation pulse of $100 \mathrm{u}$ around the expected molecular weight was applied. As can be seen in the figure, good signal-to-noise ratio $(>100: 1)$ is observed and the peptide is the base peak of the partial spectrum. If no SWIFT isolation had been applied, enkephalin would have been a minor peak in the full spectrum at these low concentrations because most of the stored ions would have originated from the chemical background (data not shown). In general, if no particular peptide mass is expected, the mass range can be subdivided into "windows" of 100-200-u width and the signal can be accumulated uritil a suitable ion population is reached. When this SWIFT preselection technique is used the detection limits on our ion trap are in the low femtomole per microliter range (typically $5-100 \mathrm{fmol}$ ).

\section{Fragmentation Performance}

The NanoES/ion trap combination offers the possibility to sequence low amounts of peptides. For clean samples $1 \mu \mathrm{L}$ of a $500-\mathrm{fmol} / \mu \mathrm{L}$ solution is sufficient to adjust instrumental parameters extensively and to ob-

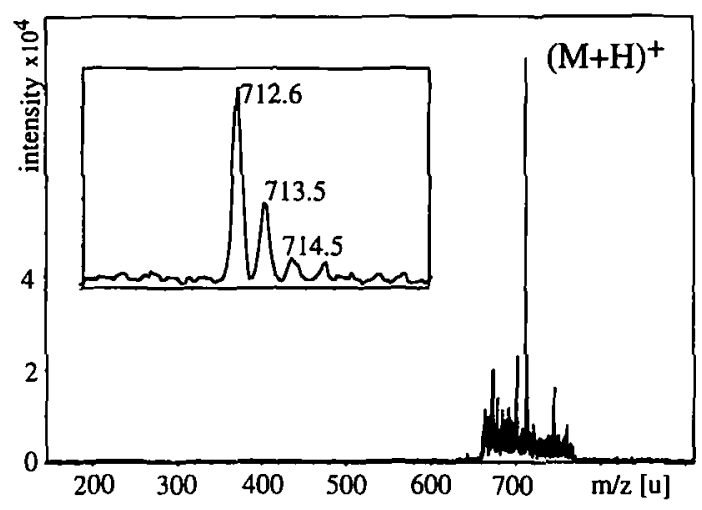

Figure 3. Spectrum of a $10-\mathrm{fmol} / \mu \mathrm{L}$ sample of leucine enkephaline-Arg. Thirty scans have been added. SWIFT pulses were used to accumulate ions from a mass range of approximately $100 \mathrm{u}$. Because only these ions were stored, the injection time could be prolonged to $4 \mathrm{~s}$.

tain at least a partial sequence of the peptide (Figures 4 and $5 \mathrm{a}$ ) by energetic collisions with the He bath gas.

Although collision-induced dissociation of peptides by resonance excitation is a standard technique for peptide sequencing in the ion trap, there are problems associated with it. The dipolar voltage signal applied to an endcap electrode causes ions with that secular frequency to undergo resonant excitation and to gain kinetic energy in the axial direction [24]. This can result in either the desired dissociation or the (undesired) ejection of the precursor ion. Ion dissociation only dominates over ejection if the pseudopotential well [25] is sufficiently deep [26] or collisions with the buffer gas remove sufficient energy to stabilize the ions [27]. An increase in the amplitude of the trapping rf frequency results in a deeper potential well, increased ion kinetic energy, and higher secular and collision frequencies.

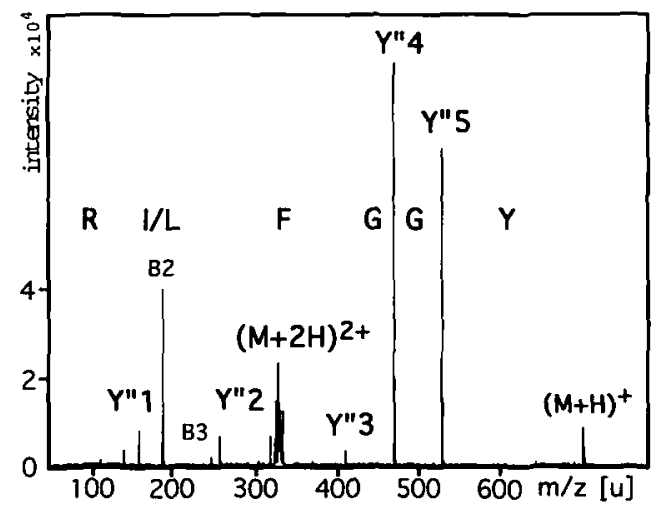

Figure 4. Fragmentation spectrum of a $500-\mathrm{fmol} / \mu \mathrm{L}$ sample of leucine enkephaline-Arg. The doubly charged ion was selected for fragmentation despite its much lower intensity compared to the single charged ion because it fragments much easier. Twenty scans have been added to obtain this spectrum. Note that the $[\mathrm{M}+\mathrm{H}]^{+}$ion is not a fragment but the result of incomplete isolation of the precursor ion. 


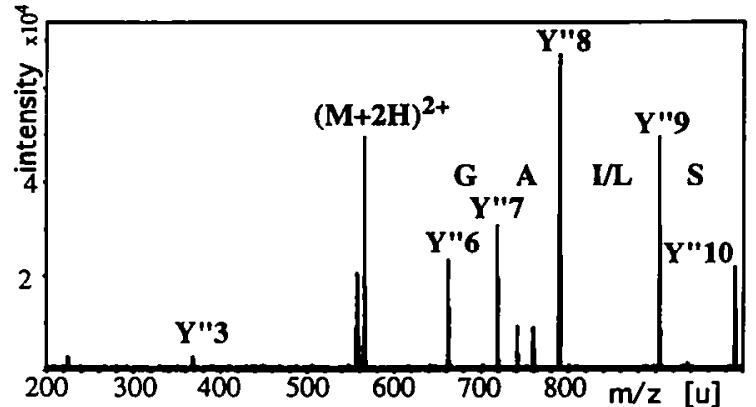

b

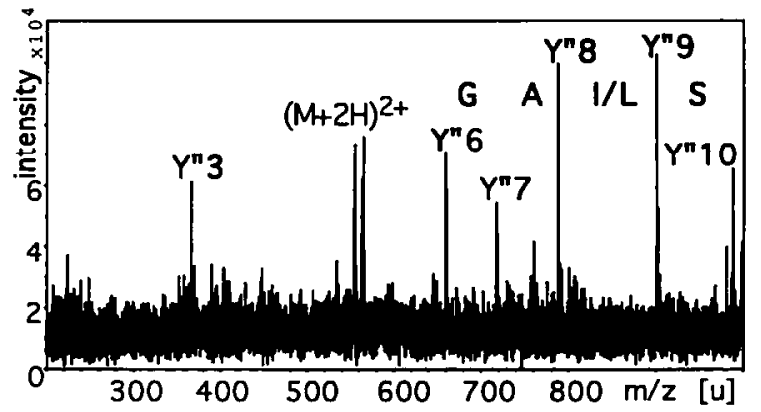

c

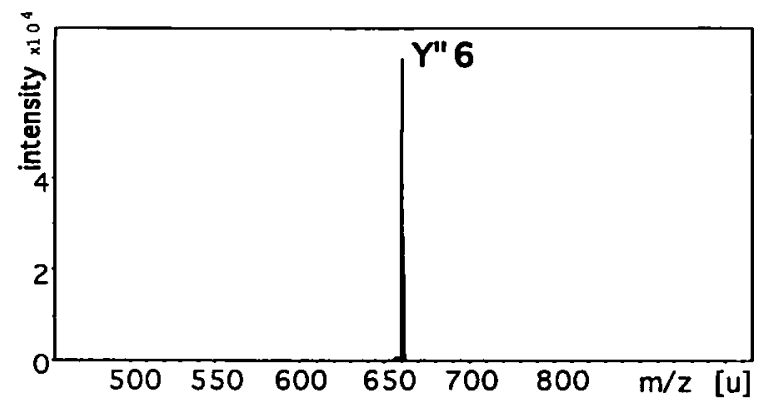

d

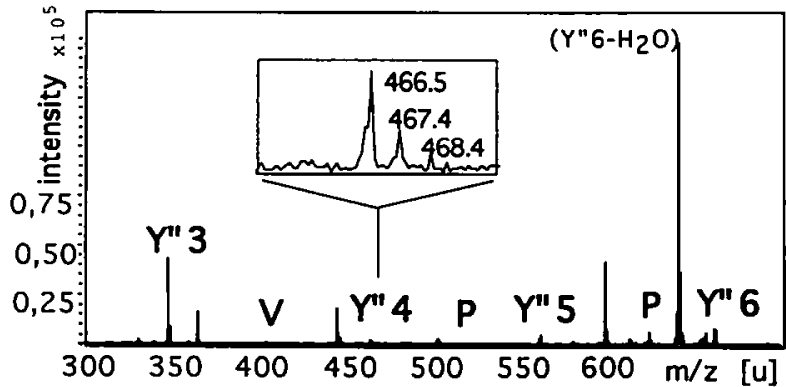

Figure 5. Fragmentation of the model peptide HSIAGPPVPPR $(5 \mathrm{pmol} / \mu \mathrm{L}$ ) by a single and two fragmentation steps. (a) The doubly charged ion was fragmented by resonance excitation that yielded a partial sequence of five amino acids. (b) The peptide was fragmented by nozzle-skimmer fragmentation. (c) SWIFT pulses were used to accumulate the $Y_{6}^{\prime \prime}$ ion in the trap. (d) The $Y_{6}^{\prime \prime}$ ion was fragmented by resonance excitation that yielded additional sequence information.

In practice a Mathieu parameter [21] of $q_{z}=0.3\left(q_{z}\right.$ is proportional to the amplitude of the trapping $\mathrm{rf}$ frequency) or higher is necessary for efficient fragmentation of peptides. Unfortunately, this limits the low mass storage range to about one third of the parent ion mass, because $q_{z}$ reaches the instability point of $q_{z}=$ 0.908 for these low mass ions $(q z$ is proportional to $m^{-1}$ ). Thus low mass fragments often do not appear in CID tandem mass spectrometry ion trap spectra of peptides.

If any partial sequence can be obtained, the peptide still can be identified in sequence databases by using the sequence tag approach [28]. Often, however, it is desirable to obtain the complete sequence. A sccond fragmentation step can circumvent the problem of low mass cutoff because the smallest fragment gives access to the lower mass range.

A second fragmentation step also is helpful because of the mechanism of ion activation in the ion trap. Fragmentation in collision cells of triple quadrupole mass spectrometers occurs on the microsecond time scale. In contrast, in the ion trap the internal energy of the precursor ions increases slowly during the typically $20-30 \mathrm{~ms}$ when the resonant excitation voltage is applied to an endcap, and the activation process stops after the dissociation because the fragment ions are off-resonance. This results in cleavage of the weakest bonds of the ion before the internal energy for higher energy pathways is reached. A second fragmentation step can be helpful because less low energy pathways are available.

If the experiment is performed by two consecutive fragmentation steps in the ion trap, a reasonable ion intensity is needed for the second fragmentation. Therefore it is, in practice, difficult to fragment minor peaks that originate from the first fragmentation step. Much higher intensities for the second fragmentation step can be realized if the first fragmentation step is performed in the interface region and a single fragment is accumulated to the space-charge limit by SWIFT. The second fragmentation step is then performed in the usual manner by resonance fragmentation.

Use of a Second Fragmentation Step in the Analysis of the Synthetic Peptide HSIAGPPVPPR

As a model case the synthetic peptide HSIAGPPVPPR was fragmented. The spectrum in Figure 5a is typical for the fragmentation behavior of doubly protonated tryptic peptides (see, for example, [29]) in that it is dominated by $Y^{\prime \prime}$ ions [30]. Intensities of the $Y^{\prime \prime}$ fragments for tryptic peptides are generally considerably larger than those of their $B$ mass complements because of the greater stability of $Y^{\prime \prime}$ ions to subsequent dissociation compared to $B$ ions, which readily dissociate to smaller fragments [29]. From the spectrum shown it is easy to determine the partial sequence $\mathrm{HS}(\mathrm{I} / \mathrm{L}) \mathrm{AG}$ by the mass differences between the $Y^{\prime \prime}$ ions. The $Y_{5}^{\prime \prime}$ and $Y_{4}^{\prime \prime}$ fragments are not visible because they correspond to the cleavage on the C-terminal side of a proline. These bonds are stable and missing fragments in the $Y^{\prime \prime}$ ion series often occur at proline or less frequently at glycine residues in the peptide chain. Therefore it is difficult to generate a complete $Y^{\prime \prime}$ series of a peptide with many prolines such as HSIAGPPVPPR. The spec- 
trum clearly shows the predominance of fragments that only need little activation energy to form. To obtain enhanced sequence information, a second fragmentation step was performed. Although the nozzle-skimmer fragmentation spectrum has poor signal-to-noise ratio (Figure 5b), SWIFT allowed the selective storage of $Y_{6}^{\prime \prime}$ fragments (Figure $5 c$ ) to a sufficient degree to generate the missing $Y_{5}^{\prime \prime}$ and $Y_{4}^{\prime \prime}$ ions in a second fragmentation step (Figure $5 \mathrm{~d}$ ). Repetition of this procedure for the $Y_{3}^{\prime \prime}$ ion resulted in the determination of the complete peptide sequence. This example shows that two steps of fragmentation can be helpful if the sequence information is limited by the preferred cleavage of weak peptide bonds and generally in the complete sequencing of "difficult" sequences. With a conventional electrospray ion source a much larger volume of sample solution would have been needed to perform these experiments.

\section{Cytochrome c Digest}

Peptide mixtures derived from sequence-specific cleavage of proteins usually are separated by HPLC before analysis either on-line or off-line in a mass spectrometer. In the case of electrospray mass spectrometry peptide separation is considered necessary because of the overlapping charge states of the different components of the mixture. The NanoES/ion trap combination should - in principle - be suited ideally to the direct analysis of unseparated mixtures because each component in the mixture can be analyzed in detail and be sequenced. As a model for this mode of operation the unseparated tryptic peptide mixture of cytochrome $c$ was analyzed. A direct analysis of the digest yielded a sequence coverage of $64 \%$ (Figure 6a). In a subsequent experiment the full mass range was divided into windows of 150-u width and ions for only these windows were stored by using SWIFT isolation during injection. The ejection of ions outside this mass range permits increased accumulation of the required ions before space-charge effects start to have a negative effect on the spectrum quality. In this way peptides that are weak in the spectrum can be measured. For the cytochrome $c$ digest the sequence coverage thus could be improved to $92 \%$.

As an example, Figure $6 \mathrm{~b}$ shows that in the SWIFT selected mass range from 380 to $500 \mathrm{u}$ five additional tryptic peptides could be detected. One of these, the peptide $(\mathrm{K} 73-\mathrm{K} 79)^{2+}$, was fragmented and a partial sequence of three amino acids could be determined from the tandem mass spectrum (Figure 7a). The $Y_{4}^{\prime \prime}$ ion of this peptide could be isolated in the nozzle-skimmer dissociation of the complete digestion mixture. After accumulation to the space-charge limit the second fragmentation step yielded the complete sequence (Figure $7 \mathrm{~b}$ ). This last experiment is justified by the sequence result (i.e., in comparison to the known sequence). In general, however, the possibility of fragmentation of other peptides in the mixture to the mass

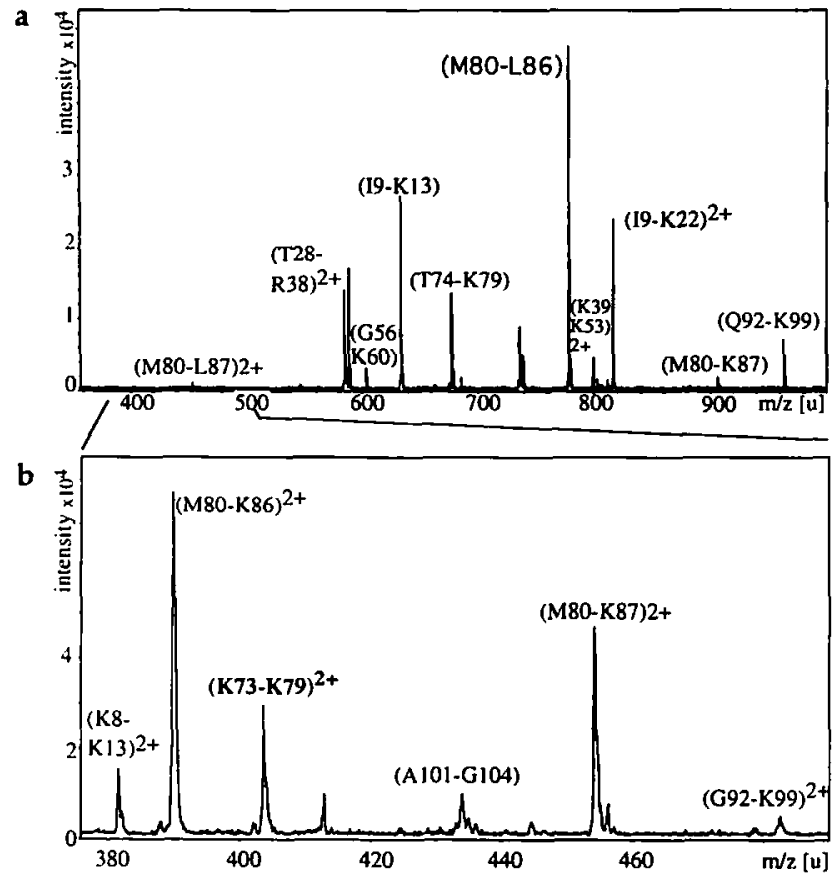

Figure 6. An unseparated cytochrome $c$ digest $(4 \mathrm{pmol} / \mu \mathrm{L})$ was analyzed by (a) direct analysis and (b) SWIFT accumulation of an approximately 150-u-wide mass range. Thirty scans were added for each of the spectra.

selected for the tandem mass spectrometric experiment must be considered.

\section{Conclusion}

NanoES coupled to the ion trap is a promising combination for the extensive investigation of complex sam-
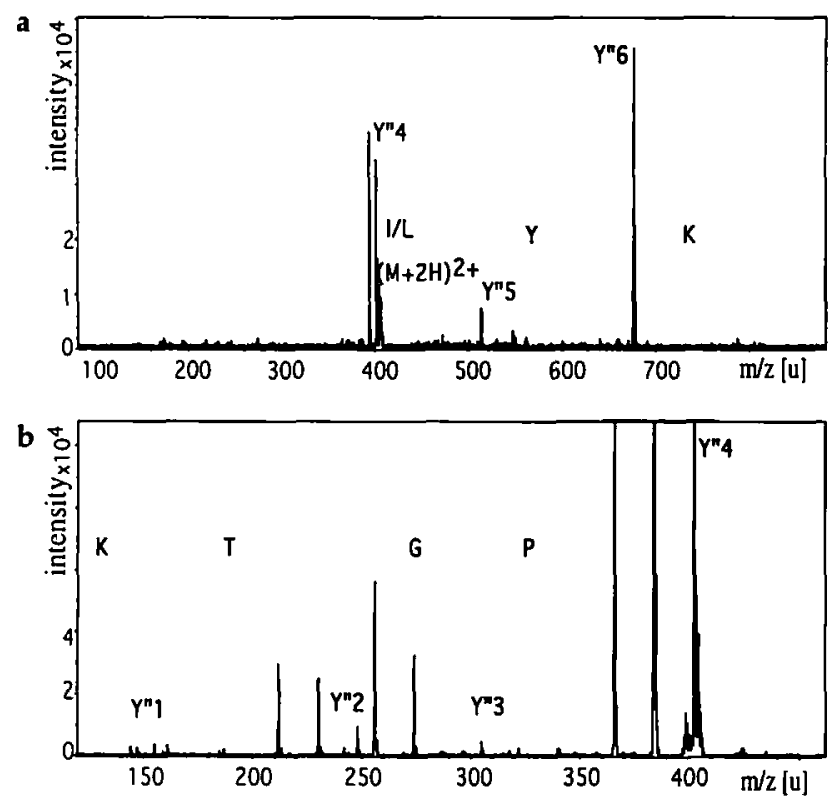

Figure 7. (a) Fragmentation of the doubly charged tryptic peptide KYPGTK (K73-K79) from the digest mixture. Fifty scans were added. (b) The unseparated digest mixture was fragmented in the interface region. The $Y_{4}^{\prime \prime}$ fragment of the peptide KYPGTK was accumulated by SWIFT and fragmented by resonance excitation. Fifty scans were added. 
ples. SWIFT preselection techniques are especially useful in this combination and can help to analyze specific components within an intense background of other ions. For isolated peptides and in special cases for peptide mixtures, the procedure that combines nozzle-skimmer fragmentation with SWIFT and a second fragmentation step that uses resonance excitation in the ion trap has the advantage of intense and well isolated precursor ions for the second fragmentation step. A novel strategy for the analysis of unseparated mixtures has been described. The mass range is "segmented" by SWIFT into ranges of, for example, $100 \mathrm{u}$, which allows the detection and fragmentation of low abundance components that otherwise might be lost through space-charge effects. All of these more complex fragmentation experiments, which may take up to half an hour for the optimization of instrumental parameters and data acquisition, can be performed on only $1 \mu \mathrm{L}$ of peptide solution by using the NanoES source. The low flow rate and the robust and stable operation of the source make it ideally suited for coupling to a trapping instruments. We and others already have coupled the NanoES source to other trapping instruments such as Fourier transform mass spectrometers [31, 32].

\section{Acknowledgments}

We thank the members of the Protein and Peptide Group of the European Molecular Biology Laboratory for their support, especially Peter Mortensen for his help in programming the SWIFT algorithm and Ole Jensen for criticism of the manuscript. Support was provided by BMBF through grant 325-4003-0310719.

\section{References}

1. Cooks, R. G.; Kaiser, R. E. Acc. Chem. Res. 1990, 23, 213-219.

2. Cooks, R. G.; Glish, G. L.; McLuckey, S. A.; Kaiser, R. E. Chem. Eng. News 1991, March 25, 26-41.

3. Loundry, F. A.; Wells, G. J.; March, R. E. Rapid Commun. Mass Spectrom. 1991, 7, 43.

4. Williams J. D.; Cox, K.; Morand, K. L.; Cooks, R. G.; Julian, R. K.; Kaiser, R. E. Proceedings of the 39th ASMS Conference on Mass Spectrometry and Allied Topics; 1991; p 1481.

5. Kaiser, R. E.; Cooks, R. G.; Syka, J. E. P.; Stafford, G. C. Rapid Commun. Mass Spectrom. 1990, 4, 30.

6. Nourse, B. D.; Cooks, R. G. Anal. Chim. Acta 1990, 228, 1.

7. Franzen, J. Int. J. Mass Spectrom. Ion Processes 1991, 106, 63-78.
8. Wang, Y.; Franzen, J. Int. J. Mass Spectrom. Ion Processes 1992, 112, 167-178.

9. Van Berkel, G. J.; Glish, G. L.; McLuckey, S. A. Anal. Chem. $1990,62,1284$.

10. Wilm, M. S.; Mann, M. Int. J. Mass Spectrom. Ion Processes 1994, 136, 167-180.

11. Demelt, H. G. Adv. Mol. Phys. 1967, 3, 53.

12. Guan, S.; Marshall, A. G. J. Am. Soc. Mass Spectrom. 1994, 5, 64-71

13. Chen, L.; Wang, T. C.; Ricca, T. L.; Marshall, A. G. Anal. Chem. 1987, 59, 454-458.

14. Julian, R. K.; Cooks, R. G. Anal. Chem. 1993, 65, 1827-1833.

15. Wahl, J. H.; Goodlett, D. R.; Udseth, H. R.; Smith, R. D. Anal. Chem. 1992, 64, 3194-3196.

16. Emmett, M. R.; Caprioli, R. M. J. Am. Soc. Mass Spectrom. 1994, 5, 605-613.

17. Schubert, M.; Mann, M.; Franzen, J.; Wang, Y. Proceedings of the 42nd ASMS Conference on Mass Spectrometry and Allied Topics; 1994; $\mathrm{p} 214$.

18. Morand, K.; Franzen, J.; Mann, M. Proceedings of the 41st ASMS Conference on Mass Spectrometry and Allied Topics; 1993; p 706.

19. Wang, Y.; Morand, K.; Mann, M.; Franzen, J.; Schubert, M. Proceedings of the 42nd ASMS Conference on Mass Spectrometry and Allied Topics; 1994; $\mathrm{p} 39$.

20. Kaiser, R. E.; Cooks, R. G.; Stafford, G. C.; Syka, J. E. P.; Hemberger, P. H. Int. I. Mass Spectrom. Ion Processes 1991, $106,79$.

21. March, K. E. Quadrupole Storage Mass Spectrometry; WileyInterscience: New York, 1989.

22. Cooley, J. W.; Tukey, J. W. Math. Comput. 1965, 19, 297-301.

23. Press, W. H.; Flannery, B. P.; Teukolsky, S. A.; Vetterling, W. T. Numerical Recipes in C; Cambridge University Press: London, 1988; Chap. 12.

24. Louris, J.; Cooks, R. G. Anal. Chem. 1987, 59, 1677-1685.

25. Major, F. G.; Dehmelt, H. G. Phys. Rev. 1968, 170, 91.

26. Charles, J.; McLuckey, S. A.; Glish, C. L. J. Am. Soc. Mass Spectrom. 1994, 5, 1031-1041.

27. Morand, K.; Cox, K.; Cooks, R. G. Rapid Commun. Mass Spectrom. 1992, 6, 520-523.

28. Mann, M.; Wilm, M. Anal. Chem. 1994, 66, 4390-4399.

29. Tang, X.; Thibault, P.; Boyd, R. K. Anal. Chem. 1993, 65, 2824-2834.

30. Roepstorff, P.; Fohlman, J. Biomed. Environ. Mass Spectrom. 1984, 11, 601.

31. Stacey, C.; Kruppa, G.; Spier, P. J.; Mann, M. Presented at the 43rd ASMS Conference on Mass Spectrometry and Allied Topics, 1995.

32. Guan, Z.; Aaserud, D.; Valaskovic, G.; Kelleher, N.; McLafferty, F. Presented at the 43rd ASMS Conference on Mass Spectrometry and Allied Topics; 1995. 